\title{
Beam Profile Measurements and PIN Diode Calibration Using the RHIC Collimators
}

R. Fliller III \& A. Drees

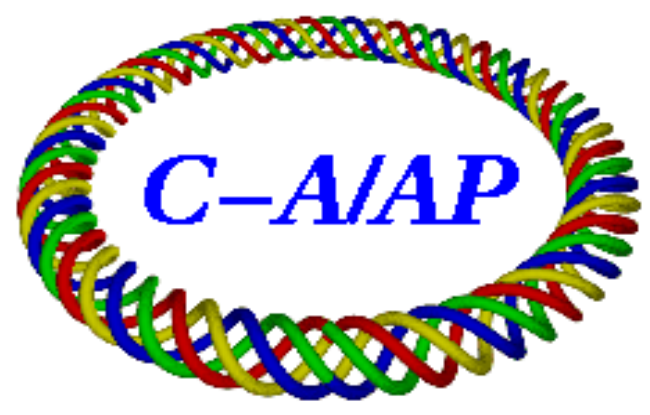

Collider-Accelerator Department Brookhaven National Laboratory Upton, NY 11973 


\title{
Beam Profile Measurements and PIN Diode Calibration Using the RHIC Collimators
}

\author{
R. Fliller III, A. Drees
}

September 13, 2004 


\begin{abstract}
By scraping away the beam using the RHIC collimators and measuring the beam current as a function of collimator position, it is possible to measure the transverse profile of the beam. This profile can then be used to obtain a rough emittance measurement. The technique is destructive and only provides an estimate of the actual emittance of the beam. Records of the count rates at the PIN diodes, may be used to calibrate the PIN diodes to a loss of beam current measured by the DCCT for low PIN diode count rates. This note presents the available data.
\end{abstract}




\section{Introduction}

Low intensity, single bunches are used during commissioning to reduce the risk of component damage. One approach to measure a beam profile is to step a collimator through the beam and measure the loss in beam current as a function of collimator position. Because this method is destructive, the measured profile is is not the true profile. However, this measurement can be useful when no other method of profile measurement is available.

In normal operation, the beam losses associated with collimation are generally small enough not to be accompanied by a noticeable additional beam current loss. However, when measuring profiles with the collimator, this is not the case. It may be possible to calibrate a PIN diode rate with a beam loss rate while measuring a beam profile with the collimator.

In this paper, we explore previous attempts at measuring beam profiles with the collimators using proton, deuteron, and gold beams in both rings of RHIC. We discuss the limits of the technique and its future usefulness. We also address the issues involved in calibrating the PIN diodes with the beam loss.

\section{Calculation of the Beam Profile from Loss Data}

The theory of measuring a beam profile using a collimator is discussed in Chapter 5 of Reference [1]. We summarize the discussion.

$f\left(x, x^{\prime}\right)$ is the one dimensional transverse phase space distribution normalized to the number of particles in the beam, $N_{0}$. The transverse beam profile in one dimension is

$$
\rho(x)=\int_{-\infty}^{+\infty} \mathrm{d} x^{\prime} f\left(x, x^{\prime}\right) .
$$

By changing variables to

$$
u^{2}=\frac{x^{2}}{\beta}+\left(\frac{\alpha}{\sqrt{\beta}} x+\sqrt{\beta} x^{\prime}\right)^{2}
$$

$f\left(x, x^{\prime}\right)$ becomes a function of $u$ alone, denoted as $\tilde{f}(u)$. Physically, $u$ is the square of the particle radius in normalized phase space. Equation 1 becomes

$$
\rho(x)=\int_{\frac{x}{\sqrt{\beta}}}^{\infty} \mathrm{d} u \frac{u \tilde{f}(u)}{\sqrt{\beta u^{2}-x^{2}}} .
$$


If a collimator is inserted to a position $x_{c}=u_{c} \sqrt{\beta}$ the number of surviving particles is

$$
N\left(u_{c}\right)=2 \pi \int_{0}^{u_{c}} u \tilde{f}(u) \mathrm{d} u .
$$

The factor of $2 \pi$ comes from an angular integral around the normalized phase ellipse. The number of particles lost by moving the collimator is

$$
\frac{\mathrm{d} N\left(u_{c}\right)}{\mathrm{d} u_{c}}=2 \pi u_{c} \tilde{f}\left(u_{c}\right) .
$$

Combining the above equation with Equation 3 gives the beam profile in terms of the particle loss

$$
\rho(x)=\frac{1}{\pi} \int_{u_{c}}^{\infty} \frac{\mathrm{d} N\left(u_{c}\right)}{\mathrm{d} u_{c}} \frac{\mathrm{d} u}{\sqrt{\beta u^{2}-x^{2}}} .
$$

By measuring the beam loss rate as a function of position, it is possible to reconstruct the beam profile using Equation 6. For a bi-gaussian beam in phase space, $\tilde{f}(u)$ is given by

$$
\tilde{f}(u)=\frac{N_{0}}{2 \pi{\sigma_{u}}^{2}} \exp \left[-\frac{u^{2}}{2 \sigma_{u}^{2}}\right] .
$$

The number of surviving particles as a function of collimator position becomes

$$
N\left(x_{c}\right)=N_{0}\left(1-\exp \left[-\frac{x^{2}}{2 \sigma_{x}^{2}}\right]\right) .
$$

where $\sigma_{x}=\sigma_{u} \sqrt{\beta}$ is the RMS beam size. Equation 6 can be solved analytically to give

$$
\begin{aligned}
\rho(x) & =\frac{N_{0}}{\sqrt{2 \pi} \sigma_{x}} \exp \left[-\frac{u^{2}}{2 \sigma_{x}^{2}}\right] \\
& =\frac{N_{0}-N(x)}{\sqrt{2 \pi} \sigma_{x}} .
\end{aligned}
$$

In this case, by making a plot of the beam current as a function of the collimator position, one can obtain the rms beam size by fitting Equation 8. For beams with a more complex distribution Equation 6 must be solved numerically.

\section{Collimator Layout}

The RHIC collimation system is shown in Figure 1. The RHIC collimation system prior to the FY2003 run consisted of a single copper scraper in each ring and 
a bent crystal collimator in the yellow ring. The scrapers are located downstream of the PHENIX detector in the drift space between the Q3 and spin rotator magnet in each ring. The yellow scraper is located in the 7 o'clock side of PHENIX and the blue scraper is located on the 8 o'clock side of PHENIX. The blue and yellow scraper are placed $47.2 \mathrm{~m}$ and $46.75 \mathrm{~m}$ downstream of the PHENIX detector respectively. The crystal collimator was not used for these measurements, but is described in References [2][3].

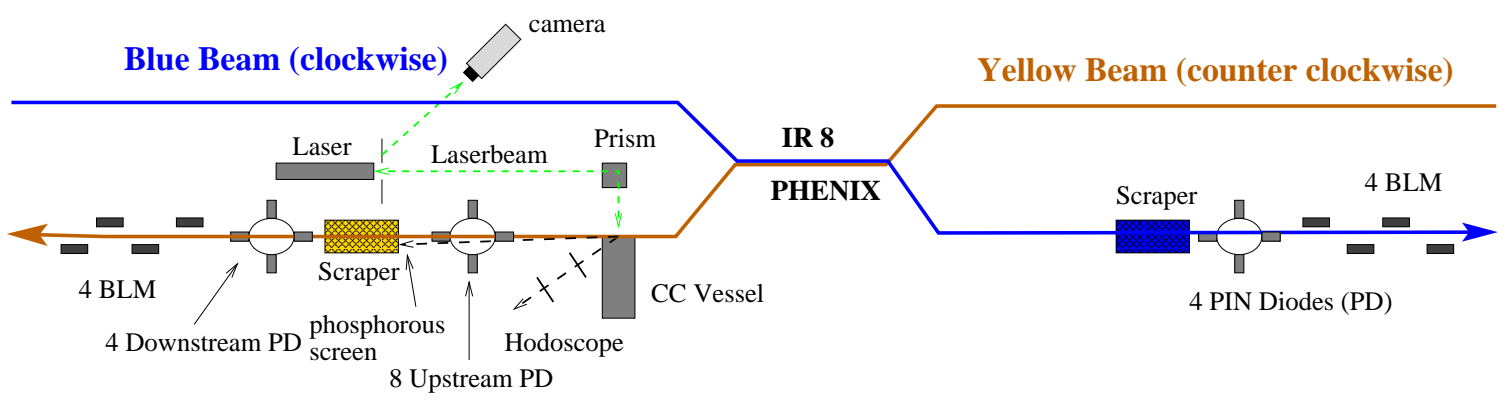

Figure 1: RHIC Collimation System. The center of RHIC is toward the bottom of the figure.

Downstream of each scraper is an array of four PD diodes, and four dedicated ionization chamber beam loss monitors (BLMs) used to measure beam losses. The diodes are Hamamatsu S3590-03 diodes used at CERN and DESY [4]. Each PD loss monitor consists of two diodes placed back to back. When a minimum ionizing particle (MIP) passes through each diode, it looses energy via ionization which generates a current pulse in the diodes. Each pulse is amplified and applied to a comparator. For simplicity, a system of two diodes in coincidence mode is referenced to as one PIN diode. The PIN diodes are very useful for measuring beam losses caused by the collimator that are too small for the BLMs to measure, as when the scraper is just touching the beam halo.

Not shown in the figure are dual plane beam position monitors (BPMs) located near the Q3 and Q4 magnets in each ring. These are used to determine the location of the beam at the collimators.

\section{Beam Profile Measurement}

Table 1 lists the existing data used to measure the beam profiles in RHIC. For each of these data sets the beam current, PIN diode rate, collimator position, and BPM readings where recorded. The data on Sept. 3, 2000, did not have the BPM readings logged. Because of a dead BPM in the blue ring and the read position shifting with beam current, the BPMs where not used in the analysis. 
Table 1: Beam Profile Data Sets

\begin{tabular}{|c|c|c|c|c|c|}
\hline Store & Ring & Species & Energy & $\sigma(\mathrm{mm})$ & $\epsilon(\pi \mathrm{mm}-\mathrm{mrad})$ \\
\hline Sept. 3, 2000 & Yellow & $\mathrm{Au}$ & Injection & $4.013 \pm 0.003$ & $11 \pm 1$ \\
02233 & Blue & $\mathrm{p}$ & Store & $5.266 \pm 0.005$ & $67 \pm 7$ \\
02320 & Yellow & $\mathrm{p}$ & Store & $3.183 \pm 0.004$ & $24 \pm 2$ \\
02320 & Blue & $\mathrm{p}$ & Store & $4.129 \pm 0.003$ & $41 \pm 4$ \\
03254 & Yellow & $\mathrm{Au}$ & Injection & $4.526 \pm 0.013$ & $16 \pm 2$ \\
03254 & Blue & $\mathrm{d}$ & Injection & $5.370 \pm 0.004$ & $22 \pm 2$ \\
03750 & Yellow & $\mathrm{p}$ & Store & $2.721 \pm 0.079$ & $18 \pm 2$ \\
03750 & Blue & $\mathrm{p}$ & Store & $1.509 \pm 0.006$ & $5.5 \pm 0.5$ \\
\hline
\end{tabular}

Each of these data sets where taken using the horizontal collimator. The vertical collimator was not used because it did not have enough travel to remove all of the beam. In each case the collimator is moved into the beam and the losses from the PIN diodes are recorded. Figure 2 shows the data from Fill 3254 in the yellow ring. The error bars are the same size as the data points. The line is a fit to Equation 8, and matches the data well. Figure 3 shows the data from Fill 2233 in the blue ring. The fit is reasonable, but does not fit the start of the loss (hence the beam tails) very well. Also notice that only $44 \%$ of the beam is removed in Figure 3 whereas $80 \%$ is scraped away in Figure 2.

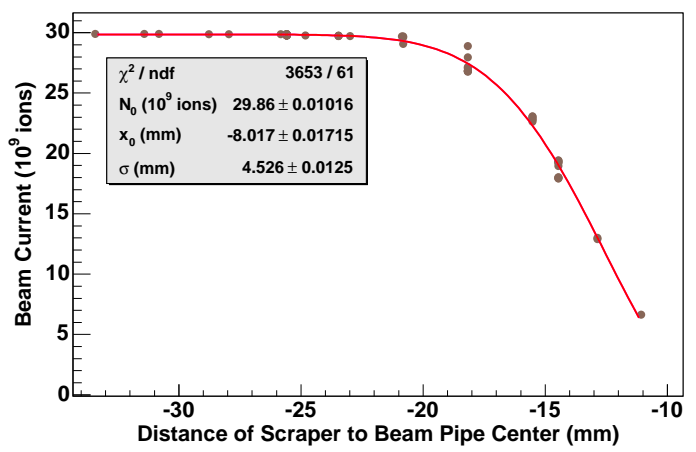

Figure 2: Data in the Yellow Ring with gold for Fill 3254

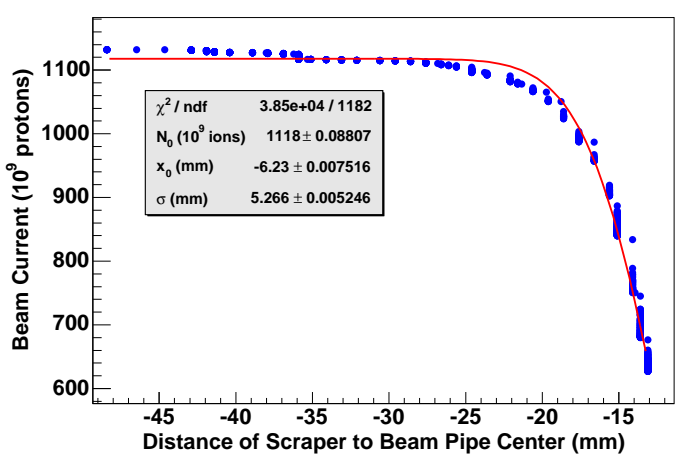

Figure 3: Data in the Blue Ring with protons for Fill 2233

The error bars on the beam size are from the fit, and the emittance error is dominated by the uncertainty in the $\beta$ function at the collimator which is approximately $10 \%$ [5]. All of the data with proton beams were taken in preparation for or during PP2PP runs to reduce the emittance of the beam. For these fills, the beams where not fully scraped away and the fits are entirely in the tails of the beam. Because the tails of the beam are broader than a gaussian, as can be seen in Figure 
3, the fitted beam size will not be very accurate. The gold and deuteron beams where fully scraped away and the emittances agree with typical values for RHIC for the gold and deuteron beams [6]. From this we note that for this technique to be successful, as much of the beam as possible needs to be removed with the scraper.

\section{PIN Diode Calibration}

Having measured the beam losses with the DCCT and the PIN diodes, it may be possible to calibrate the PIN diode rate with the current loss. Figure 4 shows a PIN diode count rate and the beam current loss as a function of time. Each time the collimator is moved, the PIN diode spikes. At the left of the graph, when the collimator is in the beam halo, the PIN diode spikes, but does not see any drop in the DCCT.

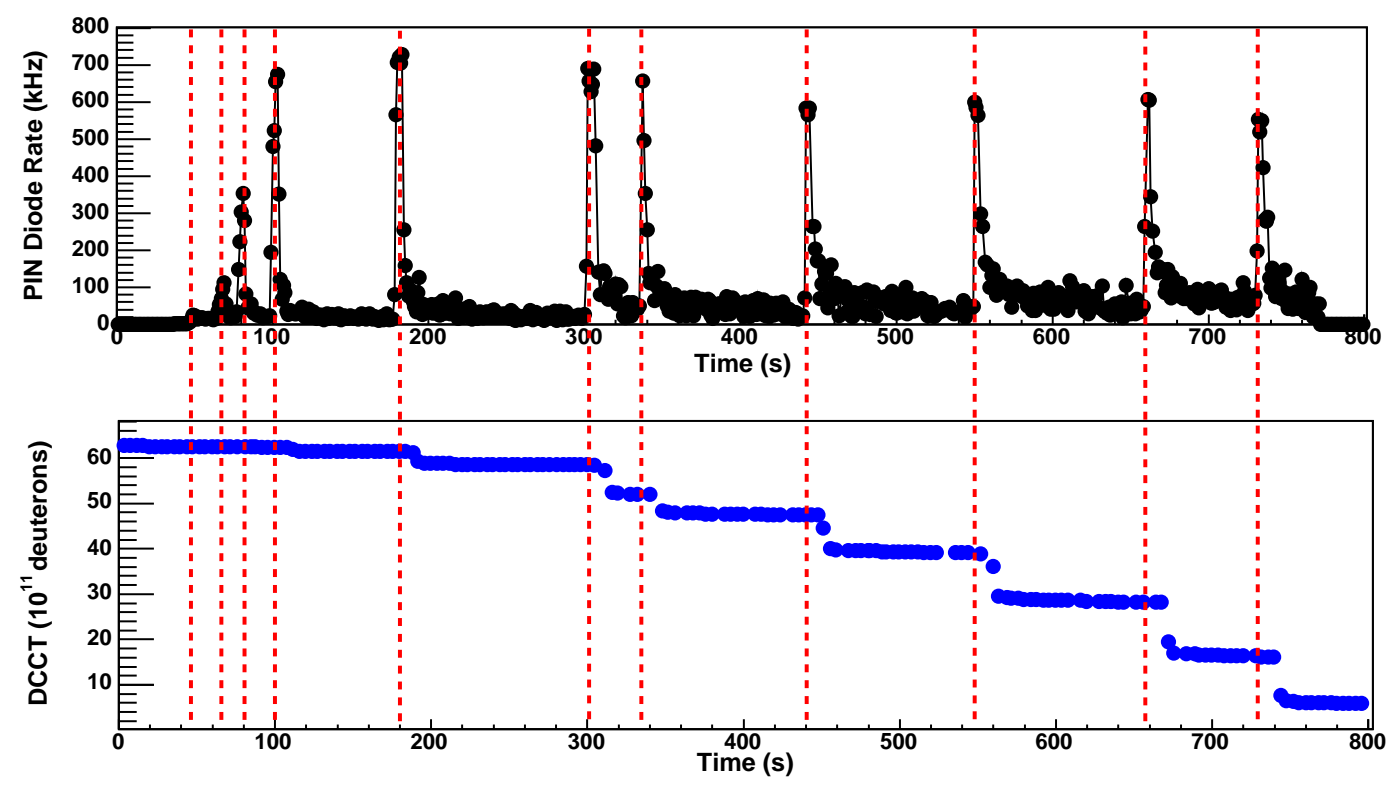

Figure 4: PIN Diode rate and Beam Current for Store 03254 in the Blue ring. The dashed lines show the times when the scraper was moved.

One may expect to calibrate the PIN diodes by equating the instantaneous rate of the diode with the slope of the beam current. This approach has two flaws. The first is that the DCCT will measure a loss anywhere in RHIC whereas the PIN diodes only see losses near the collimators. By using the collimators to remove the beam, this effect can be ignored. The second is seen in Figure 4. The drop in DCCT current happens over a few seconds after the PIN diode spike. The instantaneous PIN diode rate is not directly correlated to the DCCT slope. 

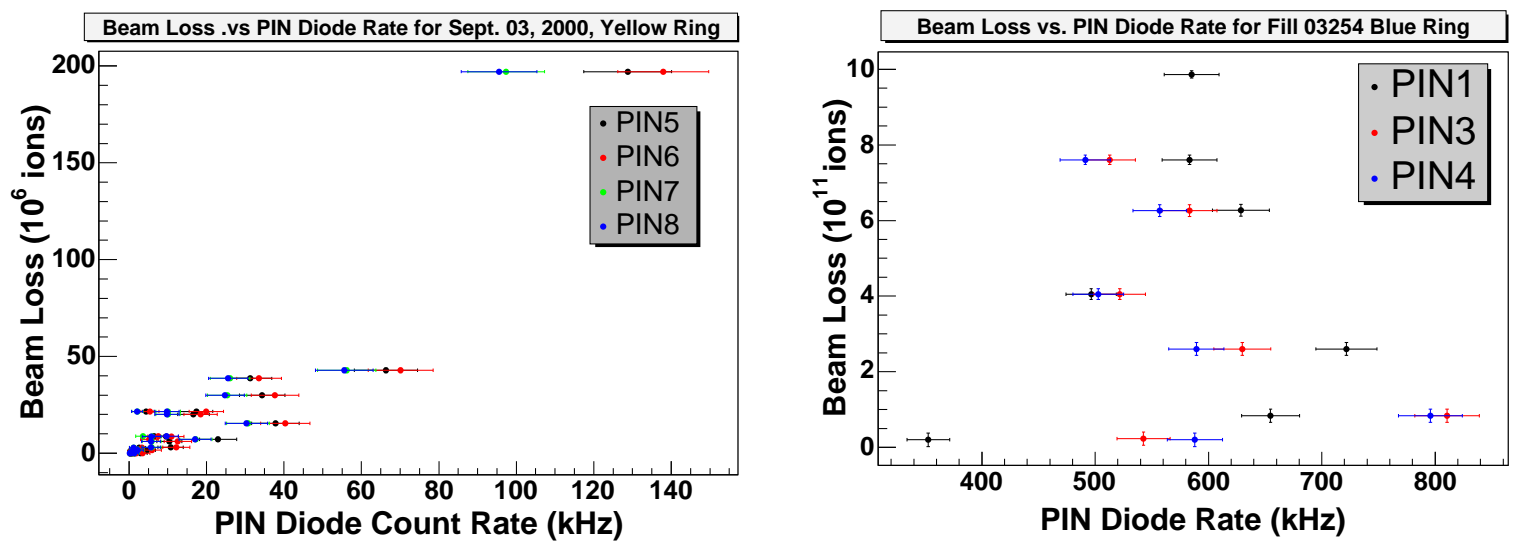

Figure 5: Beam Losses vs. PIN Diode rate for two fills.

However, another approach is to measure the height of the PIN diode spike and correlate it with the subsequent beam loss. In this way, each spike can be correlated to each drop in current. Two attempts at this are shown in Figure 5. Of all of the data sets collected, only the data taken on September 3, 2000 shows any correlation between the PIN diode loss rate and the beam losses and is shown in the left of Figure 5. This data was taken with one bunch with an intensity $0.45 \times 10^{9}$ ions. Because it is the only data set taken with one bunch, the losses where much smaller, so that the PIN diode rates stayed below $150 \mathrm{kHz}$. All other datasets had loss rates that were above this. It seems that the PIN diodes may be calibrated if they have a rate of less than $100 \mathrm{kHz}$. A linear fit is the average PIN diode rate for this fill is shown in Figure 6.

For typical PIN diode rates during collimation or experiments using the yellow collimator in the beam halo, it is possible to estimate the amount of beam being lost.

The upgrade to the collimation system will require a new calibration because the relationship between the PIN diodes and the collimator will be changed. This calibration will be most useful for the diodes that look at the primary collimator. PIN diodes for the secondary collimators will see losses causes by previous collimators as well, and a direct correlation with beam current will not be possible if other collimators are inserted. The re-calibration can be done by scraping away one bunch at a time with $10^{9}$ gold ions in it. This should be repeated a few times to cover a broader range in PIN diode rates. 


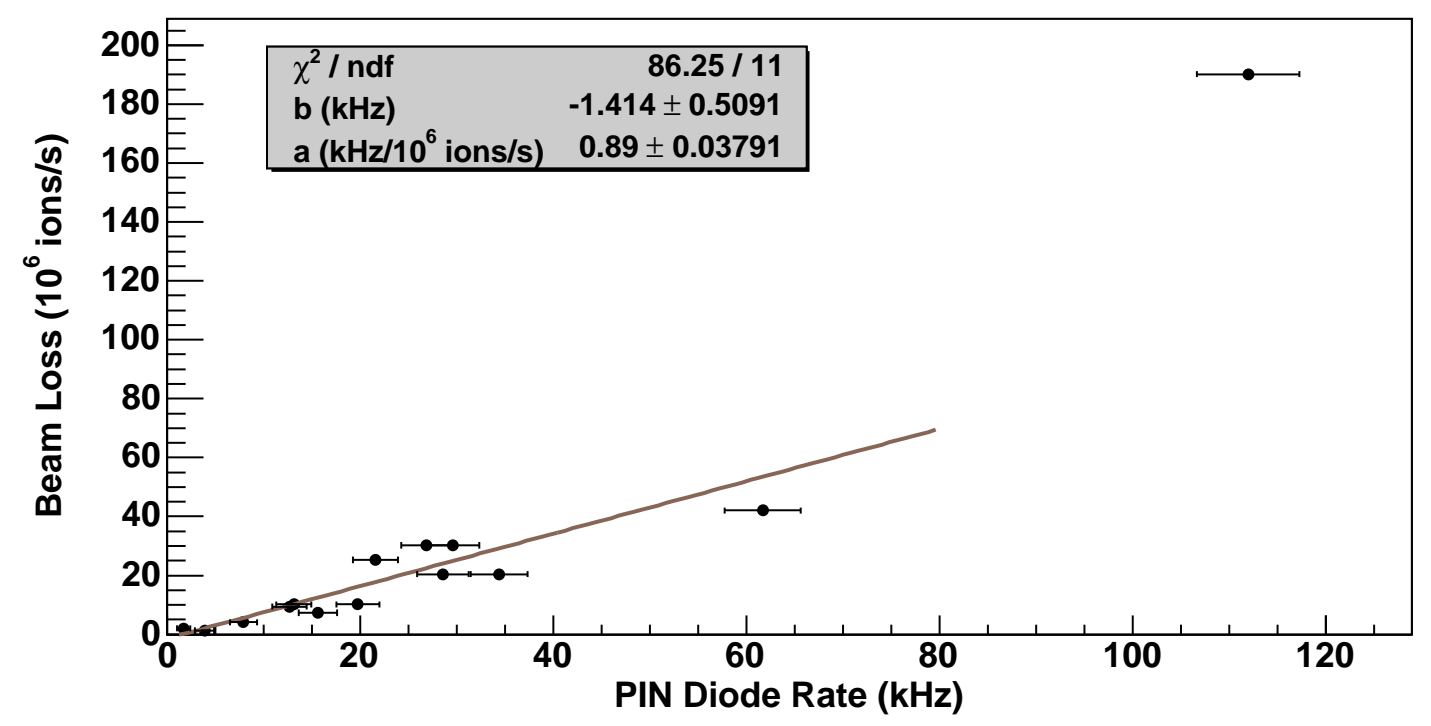

Figure 6: Linear Fit to Average PIN Diode Rate from Fill on September 3, 2000. The fit range extends the length of the line.

\section{Summary}

We have shown that scraping away an entire bunch is a viable method to measure the transverse beam profile. This can possibly be used to measure low intensity bunches, calibrate the Ionization Profile Monitors (IPMs), and provide a cross check of emittance calculations.

By scraping away a bunch, and measuring the loss of beam current, it is possible to calibrate the PIN diodes for low current losses and PIN diode rates. At higher PIN diode rates, the PIN diodes do not provide a good estimate of beam current losses. The existing calibration can be useful when doing experiments with small beam losses caused by the collimators. This calibration can be extended to slightly higher rates by scraping a high intensity bunch with the upgraded collimation system.

\section{References}

[1] M.Seidel. The Proton Collimation System of HERA. DESY 94-103, Universität Hamburg, June 1994.

[2] R. P. Fliller III, Ph. D. thesis, State University of New York at Stony Brook (to be published). 
[3] R. P. Fliller III, et. al. Crystal Collimation at RHIC Proceedings of the $29^{\text {th }}$ ICFA Advanced Advanced Beam Dynamics Workshop on Beam-Halo Dynamics, Diagnostics, and Collimation, Montauk, NY

[4] J. Bosser and G. Ferioli. Measurements of the Relative Sensitivities Between Some Beam-Loss Monitors: Part I. CERN SL/BI Note 97-01, CERN PS/BD Note 97-01, 1997.

[5] T. Satogata. Private Communication.

[6] A. Drees. Private Communication. 